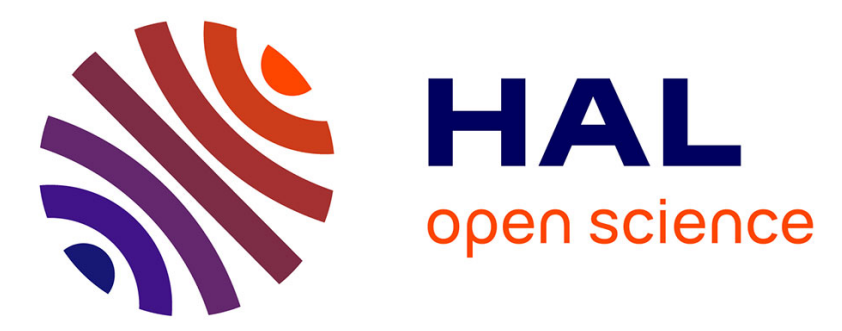

\title{
Bouc-Wen Modeling and Feedforward Control of Multivariable Hysteresis in Piezoelectric Systems: Application to a 3-DoF Piezotube Scanner.
}

Didace Habineza, Micky Rakotondrabe, Yann Gorrec

\section{- To cite this version:}

Didace Habineza, Micky Rakotondrabe, Yann Gorrec. Bouc-Wen Modeling and Feedforward Control of Multivariable Hysteresis in Piezoelectric Systems: Application to a 3-DoF Piezotube Scanner.. IEEE Transactions on Control Systems Technology, 2015, pp.1-10. hal-01303386

\author{
HAL Id: hal-01303386 \\ https://hal.science/hal-01303386
}

Submitted on 18 Apr 2016

HAL is a multi-disciplinary open access archive for the deposit and dissemination of scientific research documents, whether they are published or not. The documents may come from teaching and research institutions in France or abroad, or from public or private research centers.
L'archive ouverte pluridisciplinaire HAL, est destinée au dépôt et à la diffusion de documents scientifiques de niveau recherche, publiés ou non, émanant des établissements d'enseignement et de recherche français ou étrangers, des laboratoires publics ou privés. 


\title{
Bouc-Wen Modeling and Feedforward Control of Multivariable Hysteresis in Piezoelectric Systems: Application to a 3-DoF Piezotube Scanner
}

\author{
Didace Habineza, Student Member, IEEE, Micky Rakotondrabe, Member, IEEE \\ and Yann Le GORREC, Member, IEEE
}

\begin{abstract}
This paper is concerned with multivariable coupled hysteretic systems. The traditional Bouc-Wen monovariable hysteresis model devoted to one degrees of freedom (DoF) actuated systems is extended to model the hysteresis in systems with multiple DoF which typify strong cross-couplings. The proposed approach is able to model and to compensate for known hysteresis nonlinearities which affect smart materials. First, after presenting the new multivariable hysteresis Bouc-Wen model, a procedure of identification of its parameters is proposed. Then, we propose a multivariable compensator for the hysteresis. The compensator is based on the combination of the inverse multiplicative structure with the model which permits to avoid additional calculation of its parameters. Such advantage is essential when the number of DoF is high. All along the paper, the cases of under, over and fully actuated hysteretic systems are discussed. Finally, the proposed method is used to model and to compensate for the hysteresis in a three DoF piezoelectric tube actuator. The experimental results demonstrate its efficiency to linearize the hysteresis in the direct transfers and to minimize the hysteresis of the cross-couplings.
\end{abstract}

Index Terms - monovariable and multivariable hysteresis, classical Bouc-Wen approach, static or rate-independent hysteresis, compensation, inverse multiplicative structure, piezoelectric actuators, smart materials.

\section{INTRODUCTION}

To achieve surface or spatial positioning, applications at the micro/nano-scales such as Scanning Probe Microscopy (SPM) and Atomic Force Microscopy (AFM) require the use of actuators able to provide displacements in different directions (multi-degrees of freedom (DoF) actuators). In addition to the need of multi-DoF actuators, these applications also require high scanning speed and high bandwidth, high resolution and high precision of positioning [1]. Multi-DoF Piezoelectric based actuators (Piezotube scanners, piezo stages, etc) present many advantages for such applications: large operating bandwidth, high positioning resolution. Unfortunately, they are typified by nonlinearities. One of these nonlinearities is the hysteresis which strongly affects the final precision of positioning [2]. Even if the hysteresis in piezoelectric actuators has been widely investigated in the literature, the subject of hysteresis in multi-DoF piezoelectric actuators is quite recent. Multivariable hysteresis found in these multi-DoF actuators

The authors are with the Automatic Control and Micro-Mechatronic Systems depart., AS2M

FEMTO-ST Institute, UMR CNRS 6174 - UFC / ENSMM / UTBM

24, rue Alain Savary

25000 Besançon - France

\{didace.habineza; mrakoton; legorrec @ femto-st. fr consists of the presence of the hysteresis nonlinearity both in the expected axis (direct transfers) and in the couplings axis (cross-couplings) which poses a great challenge in their modeling, identification and control. To achieve optimal positioning accuracy, these hysteresis have to be minimized or completely suppressed.

Feedback control can be used to remove the hysteresis effects and the cross-couplings [3], [4], [5], [6], [7], [8], [9], [10], [11], but in many applications such as micropositioning, micro/nanomanipulation and micro/nanoassembly, there is a lack of usable displacement sensors to close the loop. Generally, embeddable sensors (strain gage...) do not possess the required performances while performant sensors (optical sensors...) are very expensive and bulky [2]. Some works have considered the design of piezoelectric actuators combined with integrated sensors [14], [15]. Although interesting in term of integration, these latter approaches are not valuable for already existing actuators since the systems design requires to a priori account for the sensing elements. Furthemore, adding the sensors as part of the systems will create a compromise between the performances of the actuators and of the sensors. Consequently, the performances of the actuators in such systems are less than those of purely actuated systems. Another alternative to avoid the use of external sensors is self-sensing technique which consists in exploiting piezoelectric direct and converse effects in order to estimate the actuator deflection [16]. In this, when a voltage is applied to the actuator electrodes, the actuator bends and the corresponding mechanical stress provokes the apparition of electrical charges on the actuator surface. SelfSensing consists in using an electrical circuit that amplifies these charges and transforms them into an exploitable voltage. Then, a convenient observer uses the applied voltage and the voltage provided by the electrical circuit to estimate the actuator deformation/deflection [17]. Nevertheless, this technique requires supplementary design of electronic circuit for charge amplification. Moreover, the estimation is influenced by charge leakage within the piezoelectric material which makes this technique inaccurate and inappropriate for longterm displacements measurements [18].

All these endeavors on feedback control are rendered more difficult when the number of degrees of freedom of the piezoelectric actuator is high making finally closed-loop techniques hardly implementable for micro and nano-scales in general.

Feedforward control is, in this case, an appealing alternative to feedback control in order to avoid such limitations. Two 
main approaches are used for hysteresis feedforward control. The first approach consists in precisely modeling the hysteresis and then deriving an inverse model or an approximation of the inverse. Applying this inverse model as a controller in cascade with the hysteretic piezoelectric system results in a global linearized system [2], [19], [20], [21], [22]. The second technique is the charge control compensation where, instead of inverting the hysteresis model, the input to the system is no longer the voltage, but the electric charge provided by a designed electronic circuit [23], [24], [25], [26]. This is because the relation between the charge and the output displacement is almost linear.

Different models have been used for feedforward control of hysteresis. These models can be classified into two classes. The first class is based on the superposition of several elementary hysteresis called hysterons. This class includes the Preisach techniques [27], [28], [29], [30], [31] and the PrandtlIshlinskii techniques [20], [32], [33], [34], [35]. Preisach and Prandtl-Ishlinskii approaches can be very precise if the number of hysterons is very high but, in return, become difficult to implement. The second class consists in using nonlinear differential equations as hysteresis model and as hysteresis compensator. The Bouc-Wen approach [36], [37] is in this class. The Bouc-Wen approach utilizes compact equations which are well convenient for structural analysis (stability, performances) or synthesis. Furthemore, due to the low number of parameters, the identification and the implementation of Bouc-Wen equations are facilitated.

All the above mentioned hysteresis feedforward control dealt with one-DoF piezoelectric actuators. In [40], [41] and references herein, the concept of vector Preisach hysteresis model has been studied. It consists in modeling and controlling the hysteresis in magnetic field where, instead of using scalar variables, vectors are used. The method is purely devoted to magnetic systems since the models consider the physical relation and geometrical alignment between magnetic field strength and magnetic flux density. In [42] , coupled differential equations are used to analyze the response of hysteretic structural systems under two-dimensional earthquake excitations, but the coupling effect was assigned only to some of the parameters governing the shape of hysteresis, not on the entire model parameters. Both the vector Preisach model and this latter model are devoted to 2-DoF systems. Moreover, the compensation has not been tackled.

To summarize, multivariable hysteresis modeling and compensation that consider both the direct transfers and the couplings are new and require formulation usable for different kinds of systems (piezoelectric, magnetic, magnetostrictive,...). This paper deals with the modeling, identification and feedforward control of such multivariable hysteresis, with application to piezoelectric actuators. For that, we propose to extend the classical Bouc-Wen approach, which is monovariable, to the multivariable case capable to track the hysteresis in $n$ DoF actuated systems. This is possible thanks to its low number of parameters making the identification procedure easy and realizable. To be more general, under actuated, over actuated and fully actuated hysteretic systems are investigated. Finally, in order to validate the approach, experiments on a
3-DoF piezotube scanner typified by strong hysteresis and strong cross-couplings have been carried out. The experimental results confirmed the efficiency of the proposed multivariable Bouc-Wen hysteresis modeling and control technique.

The paper is organized as follows. Section II provides a recall on the monovariable Bouc-Wen hysteresis model. Section III is devoted to the new hysteresis model: multivariable modeling with consideration of under, over and fully actuation cases. In section IV, the identification procedure of the multivariable hysteresis model is detailed. Section V deals with the derivation of the multivariable compensator that permits to cancel the hysteresis both in the direct transfers and in the couplings. The experimental application to a 3DoF piezoelectric scanner is presented in section VI. Finally, the conclusion of the paper is given in section VII.

\section{RECALL OF THE MONOVARIABLE HYSTERESIS BOUC-WEN MODEL}

The Bouc model of hysteresis [36] - further modified by Wen [37] - was initially used for nonlinear vibrational mechanics. It was afterwards widely used in structural and mechanical engineering [38]. It is defined as a set of two differential nonlinear equations relating the applied mechanical force $f$ to the deformation $x$ of a structure:

$$
\left\{\begin{array}{l}
f(x, \dot{x}, z)=\alpha k_{0} x+(1-\alpha) k_{0} z \\
\dot{z}=\dot{x}\left[A-|z|^{m}(\gamma+\beta \operatorname{sgn}(\dot{x} z))\right]
\end{array}\right.
$$

where $z$ represents an internal variable describing the structure inelastic behavior. $k_{0}$ and $\alpha$ denote the initial and the postto-pre yield stiffness respectively, and parameters $A, \gamma$ and $\beta$ control the hysteresis shape and scale.

In [39], the Bouc-Wen model represented by Eq. 1 has been re-written in order to match with piezoelectric based actuators behavior. The resulting model is given in Eq. 2. In this, the first equation relates the driving signal $U$ with the actuator displacement/deformation $y$ and with the internal state $h$. The second equation is the state-equation The piezoelectric coefficient $d_{p}$ characterizes the electromechanical transfer. Finally, the parameter $m$ of Eq. 1 was assumed to be equal to 1 due to the piezoelectric elastic structure [43].

$$
\begin{cases}y=d_{p} U-h & , y\left(t_{0}\right)=y_{0} \\ \dot{h}=A \dot{U}-\beta|\dot{U}| h-\gamma \dot{U}|h| & , h\left(t_{0}\right)=h_{0}\end{cases}
$$

The Bouc-Wen model described by (2) can be reduced in a compact notation by introducing a non-linear operator $H($. such that [39]:

$$
\left\{\begin{array}{l}
y=d_{p} U-H(U) \\
H(U)=h
\end{array}\right.
$$

with $\dot{h}=A \dot{U}-\beta|\dot{U}| h-\gamma \dot{U}|h|$.

\section{A NEW MULTIVARIABLE HYSTERESIS MODEL FOR MULTI-DOF PIEZOELECTRIC SYSTEMS}

In this section, we consider a multi-DoF piezoelectric system as presented in Fig. 1. We have $k$ inputs rassembled in a vector $U$ and $n$ outputs rassembled in a vector $y$. If $k<n$, 
the system is under actuated. If $k=n$, the system is fully actuated. And if $k>n$, the system is over actuated. When the system is under or over actuated, we say that it is rectangular. Otherwise, it is square.

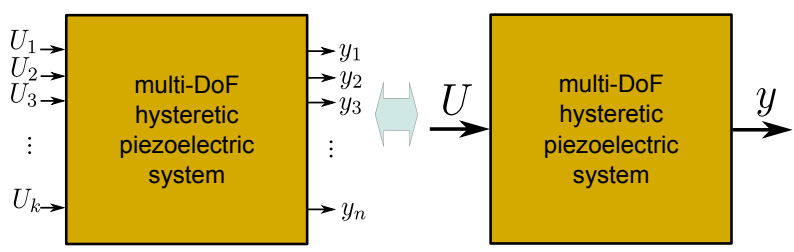

Fig. 1: A multivariable hysteretic system.

The proposition consists in extending the monovariable Bouc-Wen hysteresis model described by Eq. 2 and Eq. 3 into multivariable hysteresis. For that, the different signals and parameters are transformed into vectors and matrices. This yields ${ }^{1}$ :

$$
\left\{\begin{array}{l}
y=D U-h \\
\dot{h}=A \dot{U}-B(|\dot{\hat{U}}| \circ \hat{h})-\Gamma(\dot{\hat{U}} \circ|\hat{h}|)
\end{array}\right.
$$

equivalently:

$$
\left\{\begin{array}{l}
y=D U-H(U) \\
H(U)=h
\end{array}\right.
$$

with $\dot{h}=A \dot{U}-B(|\dot{\hat{U}}| \circ \hat{h})-\Gamma(\dot{\hat{U}} \circ|\hat{h}|)$.

In Eq. 5, $D, A, B$ and $\Gamma$ are $\mathbb{R}^{n \times k}$ matrices containing multivariable hysteresis model parameters. $U \in \mathbb{R}^{k}$ is the input voltage vector and $h \in \mathbb{R}^{n}$ is the (hysteresis) state vector. $\hat{U}$ and $\hat{h}$ are defined from $U$ and $h$ as follows:

$$
\begin{aligned}
& \dot{\hat{U}}=\left[\dot{U} ; \mathbb{O}_{(n-k) \times 1}\right] \text { and } \hat{h}=h \text { if } n>k \\
& \hat{h}=\left[h ; \mathbb{O}_{(k-n) \times 1}\right] \text { and } \dot{\hat{U}}=\dot{U} \text { if } n<k \\
& \hat{U}=U \text { and } \hat{h}=h \text { if } n=k
\end{aligned}
$$

$H(U)$ is a multivariable nonlinear operator. The compact notation in Eq. 5 will be particularly useful when synthesizing a feedforward controller in Section V.

\section{IDENTIFICATION OF THE PARAMETERS OF THE NEW MULTIVARIABLE HYSTERESIS MODEL}

Let us develop the two equations of Eq. 4 in order to derive the relation between the applied voltages $U_{j}$ with the output $y_{i}$ and with the internal state $h_{i}$. We have:

$$
\begin{gathered}
y_{i}=\left(\sum_{j=1}^{k} D_{i j} U_{j}\right)-h_{i} \\
\dot{h}_{i}=\sum_{j=1}^{k} A_{i j} \dot{U}_{j}-\sum_{j=1}^{k} B_{i j}\left|\dot{\hat{U}}_{j}\right| \hat{h}_{j}-\sum_{j=1}^{k} \Gamma_{i j} \dot{\hat{U}}_{j}\left|\hat{h}_{j}\right|
\end{gathered}
$$

According to equations Eq. 6 and Eq. 7, the parameters to be identified are $D_{i j}, A_{i j}, B_{i j}$ and $\Gamma_{i j}(1 \leq i \leq n$ and

${ }^{1}(A \circ B)$ denotes the Hadamard product of vectors $A=\left[A_{i j}\right]_{i, j}$ and $B=\left[B_{i j}\right]_{i, j}$, i.e. $(A \circ B)=\left[A_{i j} B_{i j}\right]_{i, j}$
$1 \leq j \leq k)$ which are respectively the elements of the matrices $D, A, B$ and $\Gamma$. These parameters can be real positive, negative or null. $y_{i}$ is the $i^{t h}$ output while $U_{j}$ is the $j^{t h}$ input control. Consequently, when $i=j$, we have the direct hysteresis and when $i \neq j$, we have the coupling hysteresis.

From equation Eq. 7, we observe that a signal $\dot{h}_{i}(1 \leq$ $i \leq n$ ) does not depend only on $h_{i}$ but also on $h_{j}, j$ being the subscript denoting the applied $U_{j}$. Therefore, an output $y_{i}$ $(1 \leq i \leq n)$ which naturally depends on $h_{i}$ also depends on $h_{j}$. Considering this fact, we propose the following identification procedure that can be splitted into three steps:

A) derivation of the experimental data (characterization),

B) identification of the direct hysteresis parameters,

C) and identification of the coupling hysteresis parameters.

\section{A. Experimental system characterization}

In this step, a sine or triangular input voltage $U_{j}$ (with $j=1 \cdots k)$ is applied to the piezoelectric actuator. In the meantime, the other inputs (i.e. all $U_{p}$ with $p \neq j$ and $1 \leq p \leq k)$ are set equal to zero. For each experiment, i.e. each applied $U_{j}$, the experimental data $U_{j}^{e x p}$ and $y_{i}^{e x p}$ are captured and an experimental hysteresis curve $\left(U_{j}^{\exp }, y_{i}^{\exp }\right)$ can be plotted. From equations Eq. 6 and Eq. 7, the model of such hysteresis is expressed as follows:

$$
\left\{\begin{array}{l}
y_{i}=D_{i j} U_{j}-h_{i} \\
\dot{h}_{i}=A_{i j} \dot{U}_{j}-B_{i j}\left|\dot{\hat{U}}_{j}\right| \hat{h}_{j}-\Gamma_{i j} \dot{\hat{U}}_{j}\left|\hat{h}_{j}\right|
\end{array}\right.
$$

\section{B. Identification of the parameters of the direct hysteresis equation}

This step consists in identifying the parameters of the direct hysteresis equations. From Eq. 8, the related equation is obtained by letting $i=j$ :

$$
\left\{\begin{array}{l}
y_{j}=D_{j j} U_{j}-h_{j} \\
\dot{h}_{j}=A_{j j} \dot{U}_{j}-B_{j j}\left|\dot{\hat{U}}_{j}\right| \hat{h}_{j}-\Gamma_{j j} \dot{\hat{U}}_{j}\left|\hat{h}_{j}\right|
\end{array}\right.
$$

The parameters identification is performed by using the following least square optimization problem.

Find $D_{j j}, A_{j j}, B_{j j}$ and $\Gamma_{j j}$ such that:

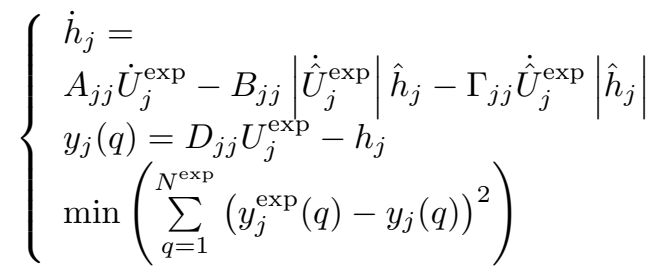

where $y_{j}^{e x p}$ and $U_{j}^{e x p}$ correspond to the experimental data of $y_{j}$ and $U_{j}$, and $N^{e x p}$ is the number of recorded points.

\section{Identification of the parameters for the couplings hysteresis equations}

Having identified the parameters of the direct hysteresis equations in the previous step, the aim of this step is to identify the remaining parameters which in fact correspond to the parameters of the couplings hysteresis equations. As we will see, the equations of the direct hysteresis in Eq. 9 
will also be used in this step. The parameters identified at step 2 are therefore used to identify the parameters $D_{i j}, A_{i j}$, $B_{i j}$ and $\Gamma_{i j}$ of the couplings hysteresis $\left(\left(U_{j}, y_{i}\right)\right.$ with $\left.i \neq j\right)$. First, remind that the equations of the couplings hysteresis are yielded from Eq. 8 by letting $i \neq j$ :

$$
\left\{\begin{array}{l}
y_{i}=D_{i j} U_{j}-h_{i} \\
\dot{h}_{i}=A_{i j} \dot{U}_{j}-B_{i j}\left|\dot{\hat{U}}_{j}\right| \hat{h}_{j}-\Gamma_{i j} \dot{\hat{U}}_{j}\left|\hat{h}_{j}\right|
\end{array}\right.
$$

As we can see from the second equation of Eq. 11, the internal state evolution $\dot{h}_{i}$ is dependant on $h_{j}$. Therefore, the state equation of $h_{j}$ given by Eq. 9 is required. The final equations for the couplings hysteresis become:

$$
\left\{\begin{array}{l}
y_{i}=D_{i j} U_{j}-h_{i} \\
\dot{h}_{i}=A_{i j} \dot{U}_{j}-B_{i j}\left|\dot{\hat{U}}_{j}\right| \hat{h}_{j}-\Gamma_{i j} \dot{\hat{U}}_{j}\left|\hat{h}_{j}\right| \\
\dot{h}_{j}=A_{j j} \dot{U}_{j}-B_{j j}\left|\dot{\hat{U}}_{j}\right| \hat{h}_{j}-\Gamma_{j j} \dot{\hat{U}}_{j}\left|\hat{h}_{j}\right|
\end{array}\right.
$$

To identify the parameters $D_{i j}, A_{i j}, B_{i j}$ and $\Gamma_{i j}$, the following least square optimization problem is used.

Find $D_{i j}, A_{i j}, B_{i j}$ and $\Gamma_{i j}$ such that:

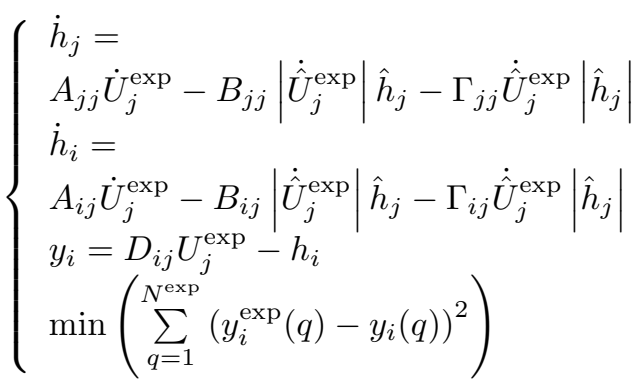

\section{FEEDFORWARd CONTROL OF MULTIVARIABLE HYSTERETIC SYSTEMS BY COMBINING THE MULTIVARIABLE BOUC-WEN MODEL WITH AN INVERSE MULTIPLICATIVE STRUCTURE}

In this section, the design of a compensator for the multivariable hysteresis is studied. We assume now that all the parameters of the multivariable Bouc-Wen model in Eq. 4 are identified. Let Fig. 2 be the system controlled by a feedforward hysteresis compensator, where $y^{\text {ref }}=\left(\begin{array}{ll}y_{1}^{r e f} & y_{2}^{\text {ref }} \cdots y_{n}^{r e f}\end{array}\right)^{T}$ is the reference input.

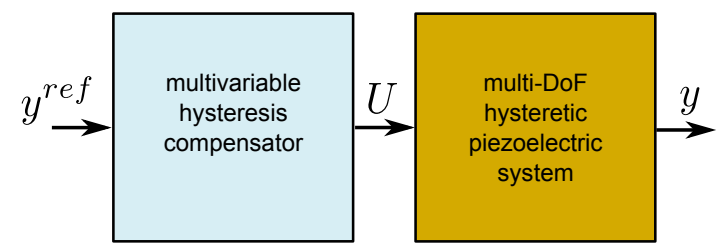

Fig. 2: Principle of the feedforward multivariable hysteresis compensation.

\section{A. Compensator equation}

The compensator is built in order to achieve the following condition:

$$
y^{r e f}=y
$$

Introducing this condition into the first equation of the multivariable model in Eq. 4, we obtain:

$$
y^{r e f}=D U-h
$$

from which we deduce the input control $U$ that satisfies the condition (14):

$$
U=D^{-1}\left(y^{r e f}+h\right)
$$

$U$ is the output of the compensator and the desired displacement $y^{\text {ref }}$ is its input.

By utilizing the compact notation (see Eq. 5), the compensator can also be written as follows:

$$
U=D^{-1}\left(y^{r e f}+H(U)\right)
$$

Fig. 3 presents the detailed block diagram of the compensator and of the multivariable Bouc-Wen hysteresis model with the compact notation. The figure shows that the structure of the compensator is inverse multiplicative. Its parameters are similar to that of the hysteresis model. This means that the compensator can be directly deduced from the identified model, and thus there is no need of additional calculation to obtain the compensator parameters. This advantage is essential in multivariable synthesis because the number of parameters increases substantially with the number of degrees of freedom. Furthemore, the proposed technique which is based on the inverse multiplicative structure permits to calculate the hysteresis compensator without an explicit calculation of the inverse model. Note that the model itself, whose the structure includes a nonlinear differential equation, is not invertible. However, the compensator calculation requires the inverse of matrix $D$ (see Eq. 17). In the next subsection, we will discuss on its invertibility and its inversion according if the system is under, over or fully actuated.

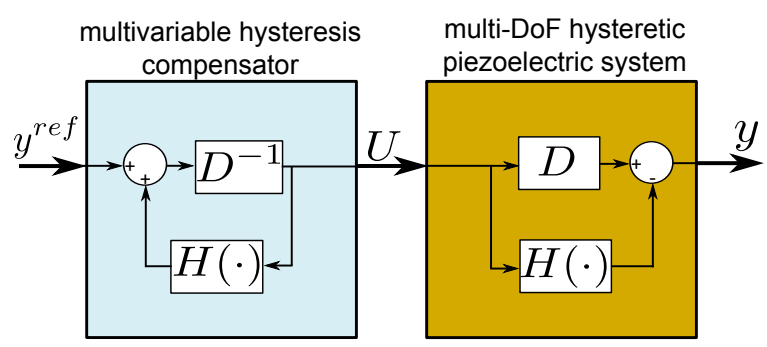

Fig. 3: Block diagram of the feedforward hysteresis control.

\section{B. Inverse of matrix $D$ and compensator implementation}

For Fully actuated systems, $D$ is square. This matrix is always invertible (non-singular) in real actuators. In fact, when designing a multi-DoF actuators, the first expectation from the designer is that each input control $U_{j}$ affects a corresponding output and axis $y_{j}$, i.e. the direct transfers $D_{j j}$ are non-null, and that the couplings are minimized. Consequently, the only case where $D$ is singular is when some of its vectors are linearly dependent. This means that when applying a voltage $U_{j}$, some or all couplings $y_{i}(i \neq j)$ exist in such a way that their sum compensates for the the output $y_{j}$. This case is very 
rare and in general, designers of actuators never allow systems having such characteristics.

For Under and Over actuated systems, $D$ is not square. In this case, we use the Moore-Penrose pseudo-inverse [44] $D^{+}$to define $U$ :

$$
U=D^{+}\left(y^{r e f}+h\right)
$$

Since $D$ is an $\mathbb{R}^{n \times k}$ matrix, we have $D^{+} \in \mathbb{R}^{k \times n}$. The MoorePenrose pseudo inverse is defined by:

$$
D^{+}=C^{T}\left(C C^{T}\right)^{-1}\left(B^{T} B\right)^{-1} B^{T}
$$

where $B \in \mathbb{R}^{n \times m}$ and $C \in \mathbb{R}^{m \times k}$ are any decomposition matrices of $D$ such that $D=B C$. The use of this pseudo inverse guarantee that the solution has a minimum 2-norm in case of over actuated systems and minimizes the 2-norm of the error in the under actuated case. The Moore-Penrose pseudo-inverse has the following properties:

$$
\left\{\begin{array}{l}
D D^{+} D=D \\
D^{+} D D^{+}=D^{+} \\
\left(D D^{+}\right)^{T}=D D^{+} \\
\left(D^{+} D\right)^{T}=D^{+} D
\end{array}\right.
$$

For under or over actuated systems, the block diagram of the compensator is similar to that of Fig. 3 but with $D^{+}$instead of $D^{-1}$.

It is also worth to notice that the structure of the operator $H(U)$ is dependent on whether the system is under, over or fully actuated. This is because the structure of $\dot{h}$ depends on whether the system is under, over or fully actuated (see Eq. 4).

\section{Application to the CONTROL of A 3-DoF PIEZOELECTRIC ACTUATOR}

This section is devoted to the application of the proposed modeling, identification procedure and feedforward control in a real case. The system to be modelled and controlled is a piezoelectric tube (piezotube) actuator able to provide displacement along 3 axes, i.e. a 3-DoF actuator.

\section{A. Experimental setup}

The actuator, pictured in Fig. 4-(a), is a piezotube scanner extensively used in atomic force microscopy (AFM). This actuator has 3-DoF and can provide displacement along three directions $(X, Y$ and $Z$ ). Based on PZT material (lead zirconate titanate), it has four external electrodes $(+\mathrm{x},-\mathrm{x}$, $+\mathrm{y}$ and $-\mathrm{y}$ ), and an inside electrode for ground (Fig. 4-(b)). When applying a positive voltage $U$ to the $+x$ electrode and the opposite voltage $-U$ to the $-\mathrm{x}$ electrode, the first sector expands while the second one contracts. This yields a global deflection (displacement) of the piezotube along the $X$ direction (Fig. 4-(c)). The same deflection can be obtained along the $Y$ direction if the voltages are applied to $+\mathrm{y}$ and $-\mathrm{y}$ electrodes. Axial deformation (extension or elongation along the $Z$ direction) is obtained by applying simultaneously $+U$ (or $-U$ ) on the four external electrodes as depicted in Fig. 4-(d). In the sequel, we simply denote $U_{x}, U_{y}$ and $U_{z}$ the voltage applied permitting to obtain the $x$, the $y$ and the $z$ displacement respectively. The piezotube used in this paper is

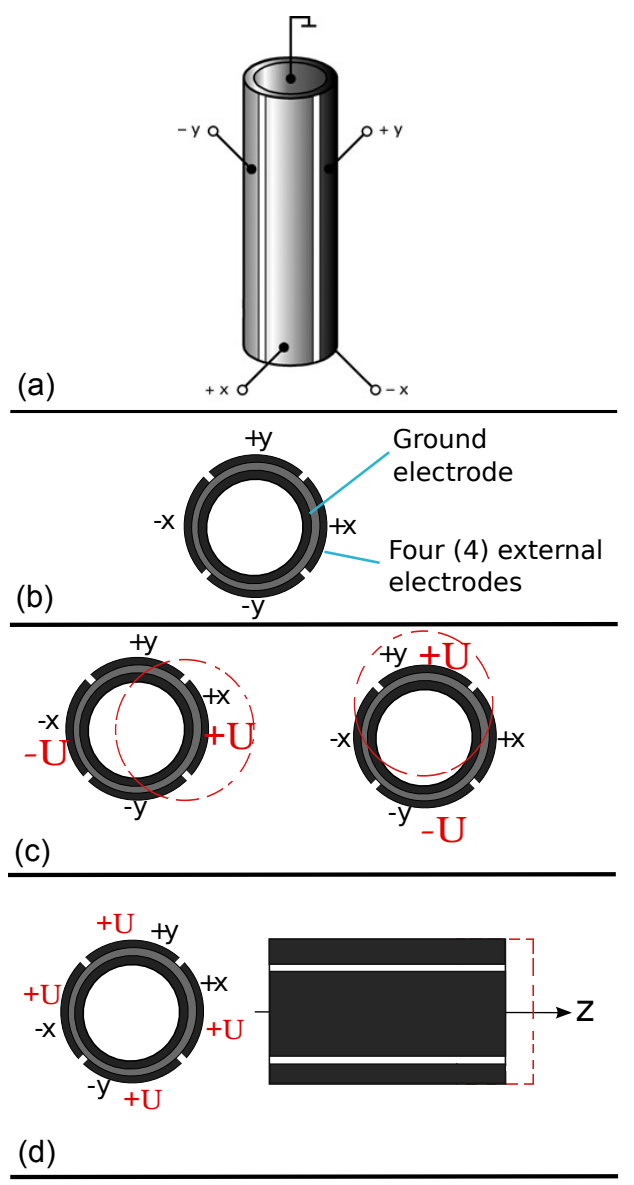

Fig. 4: (a) and (b): a piezotube actuator or scanner and its electrodes. (c) and (d): obtention of the displacements along $X, Y$ and $Z$ directions.

a PT230.94 from PIceramic company. It can be supplied with an input voltage up to $\pm 250 \mathrm{~V}$. The whole experimental setup, depicted in Fig. 5, is composed of:

- the piezotube actuator,

- a computer with Matlab-Simulink and a dSPACE board (ds1104) for the signals acquisition and for the implementation of the compensator,

- four high voltage (HV) amplifiers permitting to amplify the control signals $U_{x}, U_{y}$ and $U_{z}$,

- and three displacement sensors used to measure the displacements $x, y$ and $z$ of the actuator. The sensors, ECL202 from IBS company, are based on the inductive principle ( $E d d y$ current principle). They are set to have $40 \mathrm{~nm}$ of resolution, submicrometric accuracy, $15 \mathrm{kHz}$ of bandwidth and $\pm 250 \mu \mathrm{m}$ of range of measurement. In fact, they measure the displacement of a small cube placed at the tip of the actuator.

\section{B. Characterization, modeling and parameters identification}




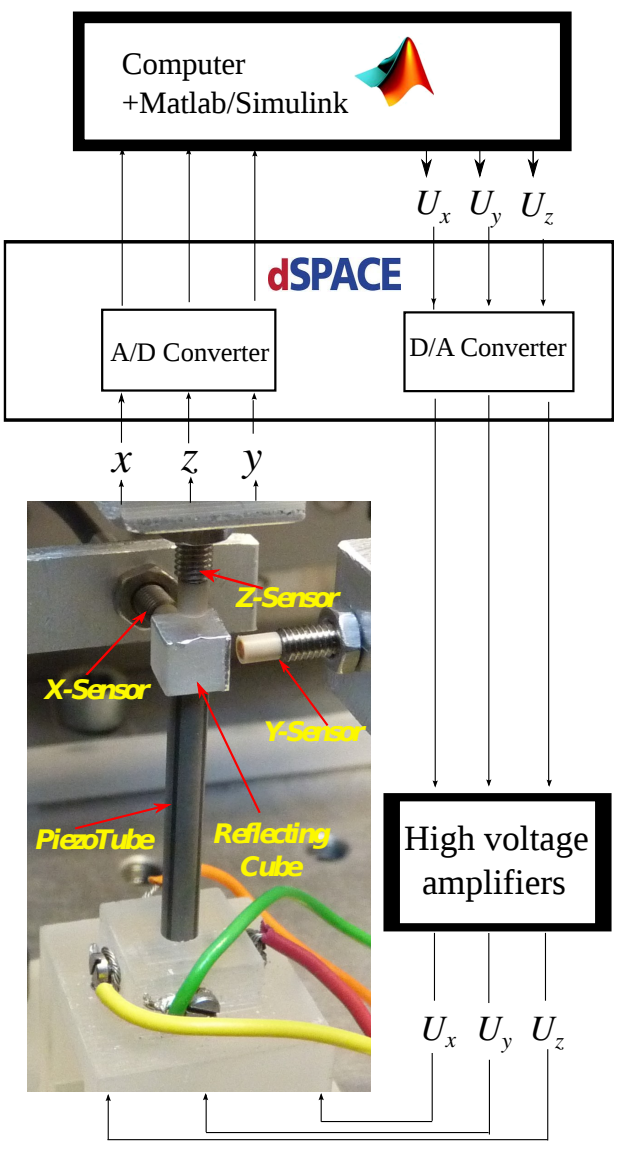

Fig. 5: Experimental setup description.

1) Multivariable hysteresis characterization: the characterization is performed as follows. A triangular voltage $U_{x}$ of amplitude $200 \mathrm{~V}$ and of frequency $0.4 \mathrm{~Hz}$ is applied to the x electrodes while both $U_{y}$ and $U_{z}$ are set to zero. The corresponding $x, y$ and $z$ displacements are pictured in Fig. 6(a), (d) and (g) respectively. Fig. 6-(a) corresponds to the direct hysteresis along $X$ axis while Fig. 6-(d) and (g) correspond to the couplings along $Y$ and $Z$ axis due to $U_{x}$. To capture the hysteresis along the $Y$ and the $Z$ axes, the same procedure than for the $X$ axis is repeated by applying $U_{y}$ and then $U_{z}$ respectively, with each time deflections $x, y$ and $z$ being captured. Fig. 6-(e) and Fig. 6-(i) are the resulting direct hysteresis for the $Y$ and for the $Z$ axis respectively. The remaining hysteresis curves are the cross-couplings.

Sine signals can also be used for the identification. The main difference relative to triangular signal is that higher dynamics (modes) are not excited. However, because triangular signals are the most used in microscopy applications, we have chosen this signal for the identification. The amplitude of the signal $( \pm 200 \mathrm{~V}$ in this case) is chosen to cover the application range. The parameters identification in a Bouc-Wen model should be done with the external loop of the hysteresis, which corresponds to the hysteresis loop obtained with the maximal range [39]. On the other hand, the frequency is chosen to be low enough. If the frequency is high, phase-lag will affect the experimental data and the pure static hysteresis found at low frequency is not anymore visible and the identified model
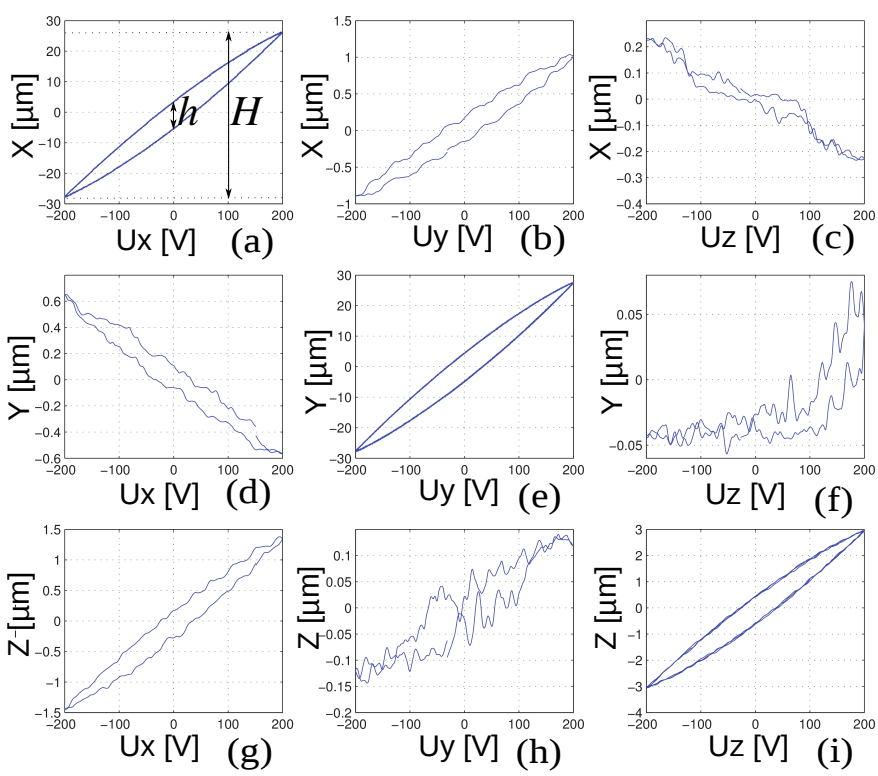

Fig. 6: Hysteresis characterization of the 3-DoF piezotube actuator.

parameters not valuable. If the frequency is too low, other nonlinearities such as creep that is out of the scope of this paper will also affect the experimental data [19]. We found that $0.4 \mathrm{~Hz}$ is a good compromise for the actuator used and for the application considered.

2) Modeling and parameters identification: The considered piezoelectric actuator is a fully actuated system with three inputs and three outputs and admits a square model. The parameters identification is carried out by using individually the nine experimental curves in Fig. 6. First, the parameters of the direct hysteresis transfers are identified through the optimization problem in Eq. 10 and considering first $j=x$, then $j=y$ and finally $j=z$ with the experimental curve of Fig. 6-(a), Fig. 6-(e) and Fig. 6-(i) respectively. The parameters of the coupling hysteresis transfers are identified through the optimization problem of Eq. 13. For instance, to identify the parameters $D_{x z}, A_{x z}, B_{x z}$ and $\Gamma_{x z}$, we use the following optimization problem:

Find $D_{x z}, A_{x z}, B_{x z}$ and $\Gamma_{x z}$ such that:

$$
\left\{\begin{array}{l}
\dot{h}_{z}= \\
A_{z z} \dot{U}_{z}^{\exp }-B_{z z}\left|\dot{\hat{U}}{ }_{z}^{\exp }\right| \hat{h}_{z}-\Gamma_{z z} \dot{\dot{U}_{z}^{\exp }}\left|\hat{h}_{z}\right| \\
\dot{h}_{z}= \\
A_{x z} \dot{U}_{z}^{\exp }-B_{x z}\left|\dot{\dot{U}_{z}^{\exp }}\right| \hat{h}_{z}-\Gamma_{x z} \dot{\hat{U}}_{z}^{\exp }\left|\hat{h}_{z}\right| \\
y_{x}=D_{x z} U_{z}^{\exp }-h_{x} \\
\min \left(\sum_{q=1}^{N^{\exp }}\left(y_{x}^{\exp }(q)-y_{x}(q)\right)^{2}\right)
\end{array}\right.
$$

where $y_{x}^{e x p}$ and $U_{z}^{e x p}$ are the experimental data of Fig. 6-(c).

After the identification procedure, we obtain the following model of the 3-DoF piezotube actuator: 

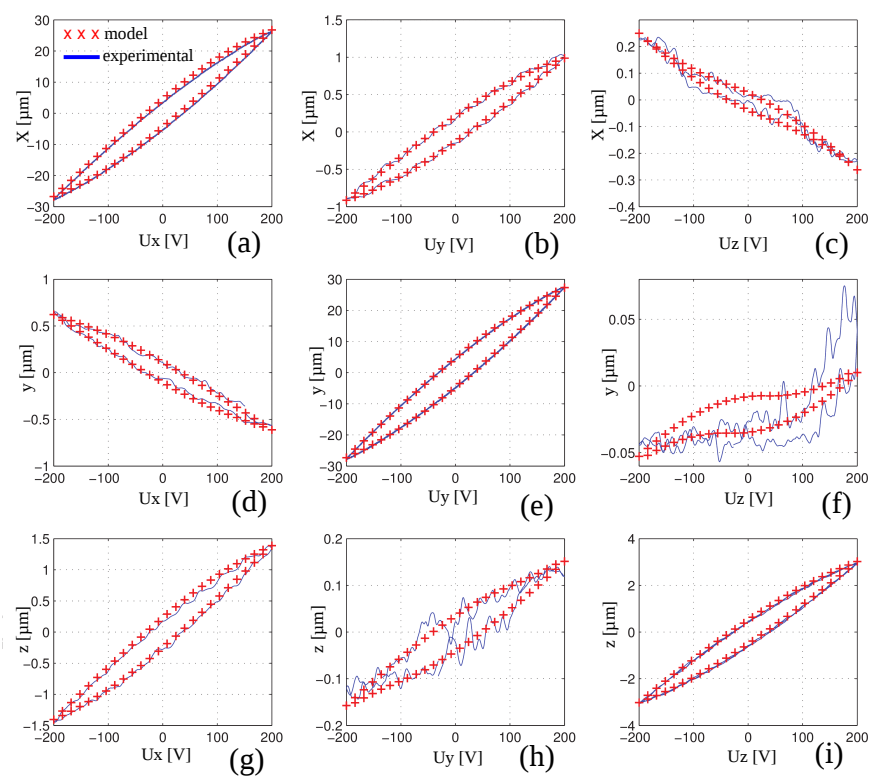

Fig. 7: Experimental curves and simulation of the identified 3-DoF Bouc-Wen model with external loops: input voltages $\pm 200 \mathrm{~V}$.

$$
\left(\begin{array}{l}
x \\
y \\
z
\end{array}\right)=\underbrace{\left(\begin{array}{ccc}
0.1663 & 0.0054 & -0.0345 \\
-0.0048 & 0.1803 & -0.0259 \\
0.0164 & -0.0360 & 0.0310
\end{array}\right)}_{D}\left(\begin{array}{l}
U_{x} \\
U_{y} \\
U_{z}
\end{array}\right)-\left(\begin{array}{l}
h_{x} \\
h_{y} \\
h_{z}
\end{array}\right)
$$

$$
\begin{aligned}
& \underbrace{\left(\begin{array}{l}
\dot{h_{x}} \\
\dot{h_{y}} \\
\dot{h_{z}}
\end{array}\right)}_{\dot{h}}=\underbrace{\left(\begin{array}{ccc}
0.0616 & 0.0013 & -0.0338 \\
-0.0026 & 0.0658 & -0.0259 \\
0.0111 & -0.0364 & 0.0160
\end{array}\right)}_{A} \underbrace{\left(\begin{array}{l}
\dot{U_{x}} \\
\dot{\dot{U}_{y}} \\
\dot{U_{z}}
\end{array}\right)}_{\dot{U}} \\
& -\underbrace{\left(\begin{array}{ccc}
0.0073 & 0.0002 & -0.0000 \\
-0.0001 & 0.0054 & 0.0000 \\
0.0004 & 0.0000 & 0.0017
\end{array}\right)}_{B} \underbrace{\left(\begin{array}{ccc}
\left|\dot{U}_{x}\right| & 0 & 0 \\
0 & \left|\dot{U}_{y}\right| & 0 \\
0 & 0 & \left|\dot{U}_{z}\right|
\end{array}\right)}_{\text {diag }(|\dot{U}|)} \underbrace{\left(\begin{array}{c}
h_{x} \\
h_{y} \\
h_{z}
\end{array}\right)}_{h} \\
& -\underbrace{\left(\begin{array}{ccc}
0.0016 & 0.0000 & -0.0003 \\
-0.0001 & 0.0010 & 0.0001 \\
0.0001 & 0.0000 & -0.0003
\end{array}\right)}_{\Gamma} \underbrace{\left(\begin{array}{ccc}
U_{x} & 0 & 0 \\
0 & U_{y} & 0 \\
0 & 0 & U_{z}
\end{array}\right)}_{\text {diag }(\dot{U})} \underbrace{\left(\begin{array}{l}
\left|h_{x}\right| \\
\left|h_{y}\right| \\
\left|h_{z}\right|
\end{array}\right)}_{|h|}
\end{aligned}
$$

3) Validation of the identified multivariable model: The simulation results from the identified model in equations Eq. 22 and Eq. 23 have been compared with the experimental results and are pictured in Fig. 7. This figure demonstrates a good agreement between the simulated and the experimental curves. We can observe that, for each curve, the error between the experimental data and the model is very small, and bounded. Nevertheless, we observe a disagreement between the experiments and the model simulation for the coupling hysteresis in Fig. 7-(f) and (h). This is due to the fact that the two experimental hysteresis are noisy and non-symmetrical,
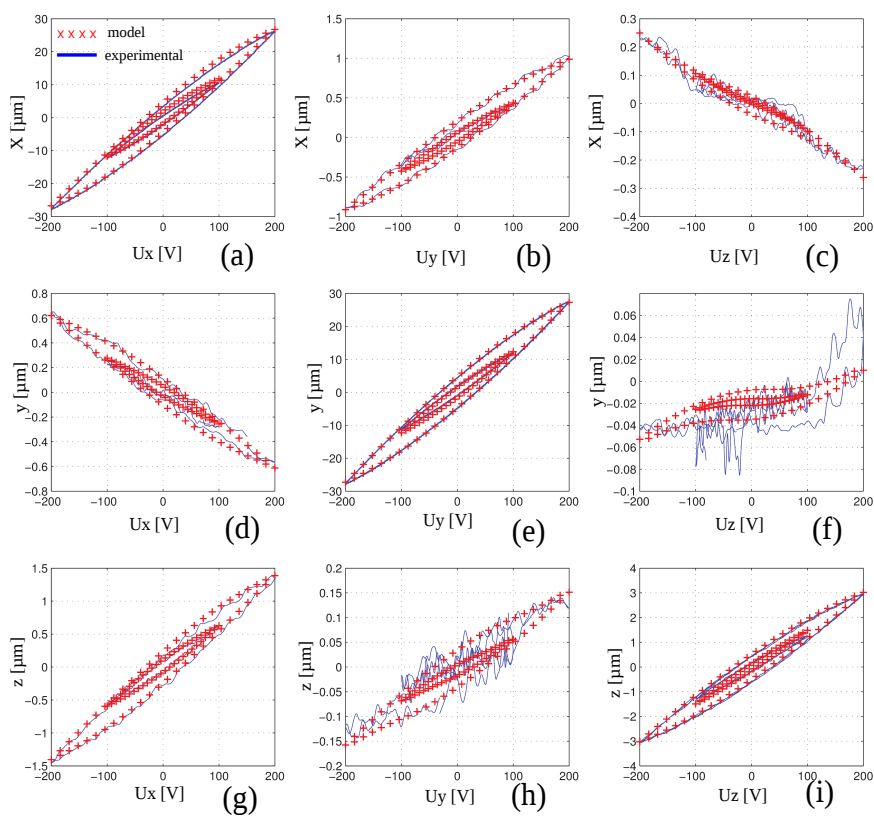

Fig. 8: Experimental curves and simulation of the identified 3DoF Bouc-Wen model with external and internal loops: input voltages $\pm 200 \mathrm{~V}$ and $\pm 100 \mathrm{~V}$.

which can not be tracked by a classical Bouc-Wen hysteresis model.

The previous validation has been effectuated with the external hysteresis loops, i.e. with input control $\pm 200 \mathrm{~V}$. To validate the model with internal loops, we also carried out experiments and model simulation with triangular input voltage ranging between $\pm 100 \mathrm{~V}$. Fig. 8 depicts the comparison between them which demonstrates that the accuracy of the identified model is still maintained. The same remark than with external loop (i.e. with $\pm 200 \mathrm{~V}$ ) is given here regarding the two cross-couplings in Fig. 8-(f) and (h) where we observe an inaccuracy due to the noise and of the asymmetry of the hysteresis.

\section{Hysteresis compensation}

We now implement the compensator of Fig. 3 by using the identified model in Eq. 22 and Eq. 23. The reference input is the vector $\left(x^{r e f}, y^{r e f}, z^{r e f}\right)^{T}$. The experiments are carried out by using triangular references of amplitude $\pm 20 \mu \mathrm{m}$ for $x^{r e f}$ and $y^{r e f}$ and $\pm 3 \mu m$ for $z^{r e f}$. Fig. 9 shows the experimental results. This figure demonstrates that the direct hysteresis have been compensated (see Fig. 9-(a), (e) and (f)) and that the cross-couplings have been reduced (rest of the figure). To more accurately evaluate the performances before and after compensation, let us use the hysteresis amplitude and the coupling amplitudes as measures. The hysteresis amplitude is defined as the ratio $\frac{h}{H}$, between the maximal residual displacement $h$ and the range of displacement $H$ as presented in Fig. 6-(a). The coupling amplitude for axis $i(i \in$ $\{X, Y, Z\})$ is defined as the ratio $\frac{H_{c p l}}{H}$ between the maximal displacement $H_{c p l}$ when a voltage $U_{j}(j \neq i)$ is applied and the maximal displacement $H$ that we would obtain with $U_{i}$. Table I summarizes the different results. They show that the 

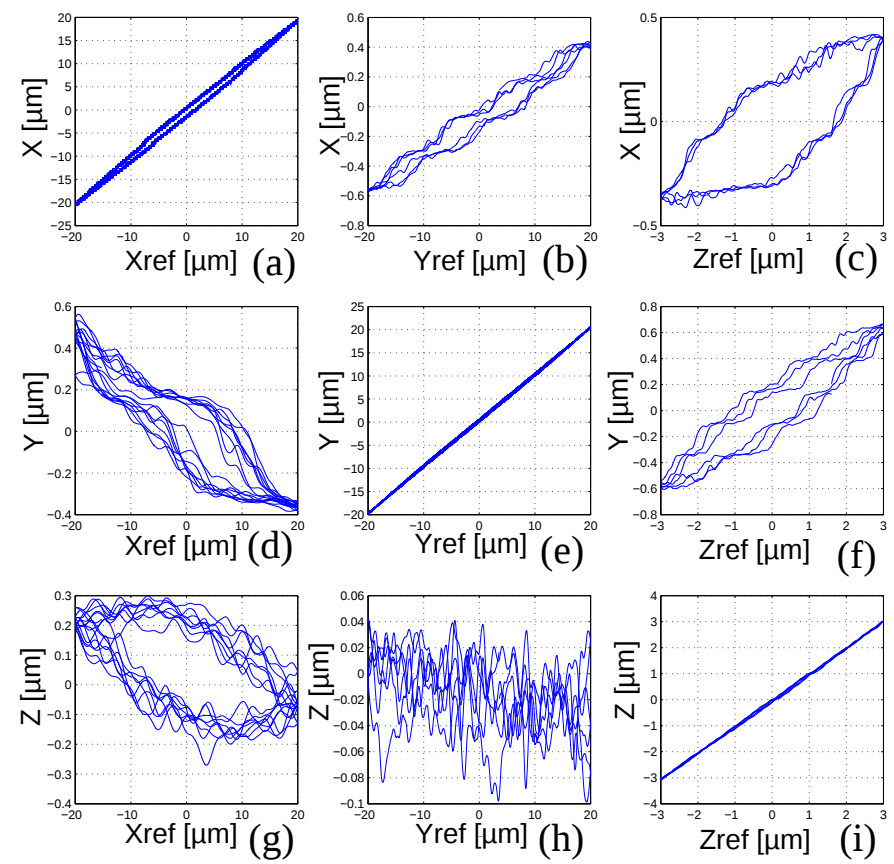

Fig. 9: Outputs $(x, y, z)^{T}$ of the compensated actuator versus the input references $\left(x^{r e f}, y^{r e f}, z^{r e f}\right)^{T}$.

direct hysteresis which were initially up to $18 \%$ have been reduced to less than $2.5 \%$ and that the cross-couplings have been substantially reduced. These verification and evaluation have been effectuated with the inductive sensors which show a noise in the measured signals. By employing optical sensors which have better measurement resolution and precision to evaluate the performaces, the accuracy of compensation can be better.

TABLE I: Comparison of the system responses, before and after compensation.

\begin{tabular}{ccc}
\hline \hline \multicolumn{3}{c}{ Before } \\
\hline $\begin{array}{c}\text { Hysteresis } \\
(h / H)\end{array}$ \\
\hline$h_{U x}^{x} / H_{U x}^{x}$ & $9.5 / 55.0=17.2 \%$ & $1.0 / 40.0=2.5 \%$ \\
$h_{U y}^{y} / H_{U y}^{y}$ & $10.1 / 56.0=18.0 \%$ & $0.0 / 40.0=0.0 \%$ \\
$h_{U z}^{z} / H_{U z}^{z}$ & $1.0 / 6.0=17.5 \%$ & $0.0 / 6.0=0.0 \%$ \\
\hline \hline Couplings & \\
amplitudes & & \\
\hline$H_{U y}^{x} / H_{U x}^{x}$ & $1.9 / 55.0=3.4 \%$ & $0.9 / 40.0=2.2 \%$ \\
$H_{U z}^{x} / H_{U x}^{x}$ & $1.9 / 55.0=3.4 \%$ & $0.8 / 40.0=2.0 \%$ \\
$H_{U x}^{y} / H_{U y}^{y}$ & $1.2 / 56.0=2.1 \%$ & $0.8 / 40.0=2.0 \%$ \\
$H_{U z}^{y} / H_{U y}^{y}$ & $0.9 / 56.0=1.6 \%$ & $1.2 / 40.0=3.0 \%$ \\
$H_{U x}^{z} / H_{U z}^{z}$ & $2.8 / 6.0=46.6 \%$ & $0.5 / 6.0=8.3 \%$ \\
$H_{U y}^{z} / H_{U z}^{z}$ & $0.3 / 6.0=5.0 \%$ & $0.0 / 6.0=0.0 \%$ \\
\hline \hline
\end{tabular}

\section{Complex trajectories tracking}

We now test the efficiency of the compensator to maintain the performances when complex reference trajectories in the space are applied. Lissajous curves have been used in [45] as novel reference signal shapes for high speed scanning probe microscopy. In this subsection, we test the efficiency of our compensator by considering Lissajous trajectory tracking in the $X-Y$ plane. The reference input is based on two sine signals: $x^{r e f}=\hat{x} \sin \left(2 \pi f_{x} t\right)$ and $y^{r e f}=\hat{y} \sin \left(2 \pi f_{y} t\right)$. The frequency ratio is set to $f_{x} / f_{y}=0.4 \mathrm{~Hz} / 0.3 \mathrm{~Hz}=4 / 3$ and the amplitudes $\hat{x}$ and $\hat{y}$ are both set to $20 \mu \mathrm{m}$. Fig. 10-(a), (b) and (c) show the time domain, the spatial curves and the tracking errors respectively. In order to evaluate the gained performaces, the outputs of the actuator without compensator are also pictured in the same figures. For this latter case, the input control $U_{x}$ and $U_{y}$ have been scaled with a gain of $\frac{20 \mu \mathrm{m}}{150 \mathrm{~V}}$ in order to make the comparison possible, which comes down to a linear feedforward control.

From these figures, we notice the substantial improvement of the tracking when the proposed multivariable hysteresis compensator is applied. This improvement is also seen in Fig. 10-(c) by comparing the tracking errors with and without the proposed compensator, for both $X$ and $Y$ axis. The experiments were carried out at different fixed values of $Z$ and they demonstrate the same performaces.

\section{CONCLUSIONS}

This paper dealt with the modeling, the identification procedure and the compensation of hysteresis in multi-DoF actuated systems. Such systems exhibit hysteresis both in the direct transfers and in the couplings axes. The proposed model consists in extending the classical Bouc-Wen hysteresis model devoted to one-DoF systems. We have shown that this model can be efficiently used for control purpose by using an inverse multiplicative structure feedforward strategy. This technique permits to avoid additional controller parameters computation. Such advantage is essential when the number of DoF is high. To validate the proposed modeling and compensation, we applied the method to a three DoF piezoelectric tube actuator. The experimental results demonstrated the substantial reduction of the hysteresis in the three axis and of the couplings of the controlled actuator. Future work will focus on the adaptation of the proposed approach in order to take into account the modeling and the compensation of rate dependent hysteretic systems.

\section{ACKNOWLEDGMENT}

This work is supported by the national ANR-JCJC CMUMS-project (National young investigator project ANR-12JS03007.01: Control of Multivariable Piezoelectric Microsystems with Minimization of Sensors).

\section{REFERENCES}

[1] K. K. Leang, Z. Qingze, and D. Santosh, "A review of feedforward control approaches in nanopositioning for high-speed SPM", Journal of dynamic systems, measurement, and control, vol. 131, no. 6, pp. 0611011-061101-19, Nov. 2009.

[2] S. Devasia, E. Eleftheriou, and S. O. R. Moheimani, "A survey of Control Issues in Nanopositioning", IEEE Transactions on Control Systems Technology, vol. 15, no. 5, pp. 802-823, Sept. 2007.

[3] Y. K. Yong, S. S. Aphale, and S. O. R. Moheimani, "Design, identification and control of a flexure-based X-Y stage for fast nanoscale positioning", IEEE Transactions on Nanotechnology, vol. 8, no. 1, pp. 46-54, Jan. 2009. 

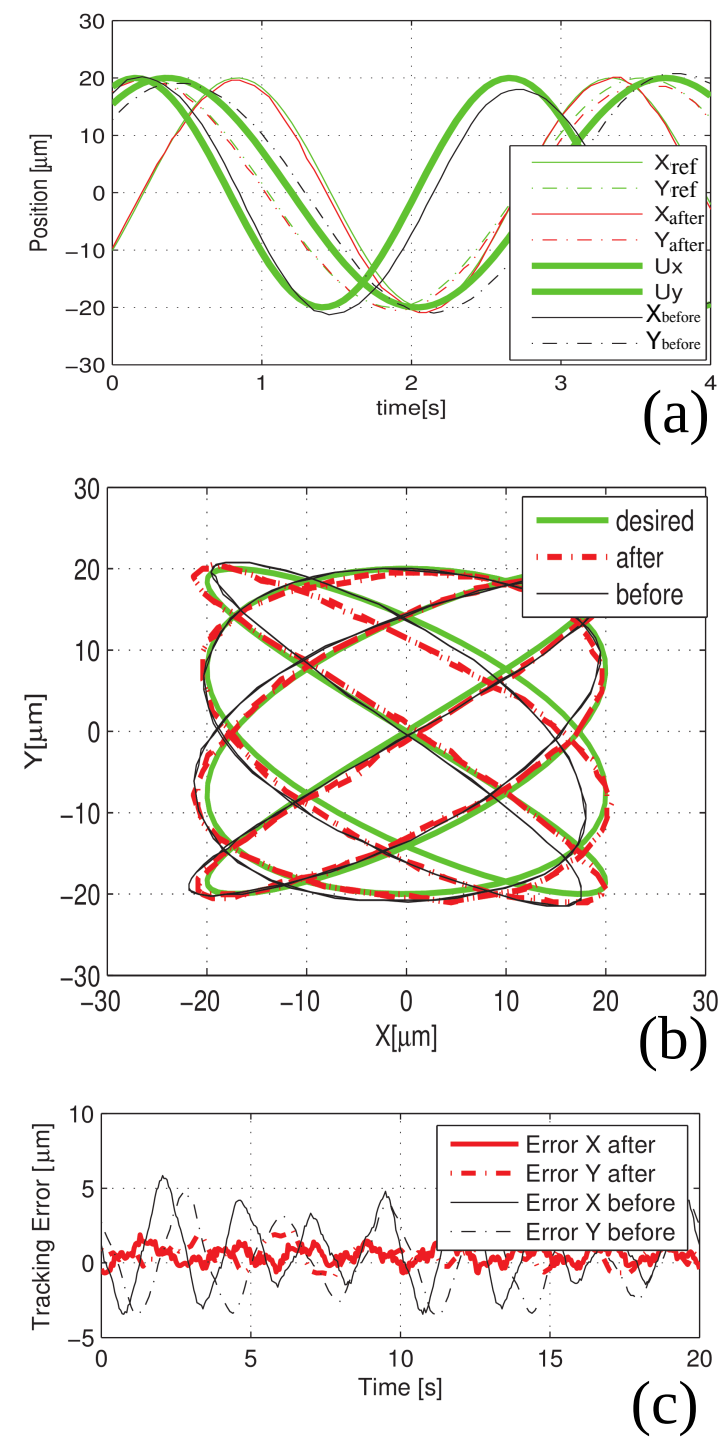

Fig. 10: Lissajous curves tracking before and after compensation. (a): outputs $x$ and $y$ versus the time. (b): spatial curves. (c): tracking error.

[4] B. J. Kenton and K. K. Leang, "Design and control of a three-axis serialkinematic high-bandwidth nanopositioner", IEEE/ASME Transactions on Mechatronics, vol. 17, no. 2, pp. 356-369, Apr. 2012.

[5] G. Schitter, K. J. Astrom, B. E. DeMartini, P. J. Thurner, K. L. Turner, and P. K. Hansma, "Design and modeling of a high-speed AFM scanner", IEEE Transactions on Control Systems Technology, vol. 15, no. 5, pp. 906-915, Sept. 2007.

[6] I. Mahmood, K. Liu, and S. O. R. Moheimani, "Two sensor based Hinf control of a piezoelectric tube scanner", Proceedings of the 17th World Congress, pp. 8251-8256, Seoul, Korea, Jul. 2008.

[7] Q. Xu and Y. Li, "Dahl model-based hysteresis compensation and precise positioning control of an X-Y parallel micromanipulator with piezoelectric actuation", Journal of dynamic systems, measurement and control, vol. 132, no. 4, pp. 041011-1-041011-12, Jun. 2010.

[8] A. Bazaei, Y. K. Yong, S. O. R. Moheimani, and A. Sebastian, "Tracking of Triangular References Using Signal Transformation for Control of a Novel AFM Scanner Stage", IEEE Transactions on Control Systems Technology, vol. 20, no. 2, pp. 453-464, Mar. 2012.

[9] S. Salapaka, A. Sebastian, J. P. Cleveland, and M. V. Salapaka, "High bandwidth nano-positioner: A robust control approach", Review of scientific instruments, vol. 73, no. 9, pp. 3232-3241, Sept. 2002.

[10] H. Habibullah, H. R. Pota, I. R. Petersen, M. S. Rana, "Reduction of cross-coupling between $\mathrm{X}-\mathrm{Y}$ axes of piezoelectric scanner stage of atomic force microscope for faster scanning", in Proceedings of IEEE International Conference on Control Applications, Aug. 2013, pp. 455460.

[11] N. Tamer and M. Dahleh, "Feedback control of piezoelectric tube scanners", in Proceedings of the 33rd IEEE Conference on Decision and Control, Dec. 1994, pp. 1826-1831.

[12] M. Rakotondrabe, Y. Haddab and P. Lutz, "Quadrilateral modelling and robust control of a nonlinear piezoelectric cantilever", IEEE Transactions on Control Systems Technology, vol. 17, no. 3, pp. 528-539, May 2009.

[13] S. Khadraoui, M. Rakotondrabe and P. Lutz, "Interval Modeling and Robust Control of Piezoelectric Microactuators", IEEE Transactions on Control Systems Technology, vol. 20, no. 2, pp. 486-494, Mar. 2012.

[14] R. G. Ballas, "Piezoelectric multilayer beam bending actuators: Static and dynamic behavior and aspects of sensor integration", Springer, 2007.

[15] A. J. Fleming, and K. K. Leang, "Integrated strain and force feedback for high-performance control of piezoelectric actuators", Sensors and Actuators, vol. 161, no. 1, pp. 256-265, Jun. 2010.

[16] S. O. R. Moheimani, and A. J. Fleming, "Piezoelectric transducers for vibration control and damping", Springer, 2006.

[17] M. Rakotondrabe, "Combining self-sensing with an unkown-inputobserver to estimate the displacement, the force and the state in piezoelectric cantilevered actuators", in Proceedings of American Control Conference, Jun. 2013, pp. 4516-4523.

[18] I. A. Ivan, M. Rakotondrabe, P. Lutz, and N. Chaillet, "Quasistatic displacement self-sensing method for cantilevered piezoelectric actuators", Review of Scientific instruments, vol. 80, no. 6, pp. 065102-1-065102-8, Jun. 2009.

[19] M. Rakotondrabe, C. Clévy and P. Lutz, "Complete open loop control of hysteretic, creeped and oscillating piezoelectric cantilever", IEEE Transactions on Automation Science and Engineering, vol. 7, no. 3, pp. 440-450, Jul. 2010.

[20] K. Kuhnen and H. Janocha, "Inverse feedforward controller for complex hysteretic nonlinearities in smart-material systems", Control and Intelligent Systems, vol. 29, no. 3, pp. 74-83, 2001.

[21] K. K. Leang, Qingze Zou, and S. Devasia, "Feedforward control of piezoactuators in atomic force microscope systems", IEEE Control Systems, vol. 29, no. 1, pp. 70-82, Feb. 2009.

[22] H. Jung, J. Y. Shim, and D. Gweon, "New open-loop actuating method of piezoelectric actuators for removing hysteresis and creep", Review of Scientific Instruments, vol. 71, no. 9, pp. 3436-3440, Jun. 2000.

[23] G. M. Clayton, S. Tien, A. J. Fleming, S. O. R. Moheimani and S. Devasia, "Hysteresis and vibration compensation in piezoelectric actuators by integrating charge control and inverse feedforward", 4th IFAC Symposium on Mechatronic Systems, pp. 812-818, Heidelberg, Germany, Sept. 2006.

[24] J. A. Main, E. Garcia, and D. V. Newton, "Precision position control of piezoelectric actuators using charge feedback", Journal of Guidance, control, and dynamics, vol. 18, no. 5, pp. 1068-1073, Oct. 1995.

[25] S. O. R. Moheimani, and B. J. G. Vautier, "Resonant control of structural vibration using charge-driven piezoelectric actuators", in Proceedings of IEEE Conference on Decision and Control, Dec. 2004, pp. 1021-1035.

[26] B. Bhikkaji, M. Ratnam, A. J. Fleming, and S. O. R. Moheimani, "High performance control of piezoelectric tube scanners", IEEE Transactions on Control Systems Technology, vol. 15, no. 5, pp. 853-866, Sept. 2007.

[27] M. J. Jang, C. L. Chen, and J. R. Lee, "Modeling and control of a piezoelectric actuator driven system with asymmetric hysteresis", Journal of the Franklin Institute, Elsevier, vol. 346, no. 1, pp. 17-32, Feb. 2009.

[28] G. Song, Jinqiang Zhao, Xiaoqin Zhou, J. A. De Abreu-Garcia, "Tracking control of a piezoceramic actuator with hysteresis compensation using inverse Preisach model", IEEE/ASME Transactions on Mechatronics, vol. 10, no. 2, pp. 198-209, Apr. 2005.

[29] H. Hu, and R. Ben Mrad, "On the classical Preisach model for hysteresis in piezoceramic actuators", Mechatronics, vol. 13, no. 2, pp. 85-94, Mar. 2002.

[30] Ping Ge, and M. Jouaneh, "Generalized Preisach model for hysteresis nonlinearity of piezoceramic actuators", Precision Engineering, vol. 20, no. 2, pp. 99-111, Mar. 1997.

[31] R. V. Iyer, X. Tan, P. S. Krishnaprasad, "Approximate inversion of the Preisach hysteresis operator with application to control of smart actuators", IEEE Transactions on Automatic Control, vol. 50, no. 6, pp. 798-810, Jun. 2005.

[32] M. Rakotondrabe, "Classical Prandtl-Ishlinskii modeling and inverse multiplicative structure to compensate hysteresis in piezoactuators", in Proceedings of American Control Conference, Montreal, Canada, Jun. 2012, pp. 1646-1651. 
[33] Wei Tech Ang, P. K. Khosla, and C. N. Riviere, "Feedforward controller with inverse rate-dependent model for piezoelectric actuators in trajectory-tracking applications", IEEE/ASME Transactions on Mechatronics, vol. 12, no. 2, pp. 134-142, Apr. 2007.

[34] M. Al Janaideh, S. Rakheja, and Chun-Yi Su, "An analytical generalized Prandtl-Ishlinskii model inversion for hysteresis compensation in micropositioning control", IEEE/ASME Transactions on Mechatronics, vol. 16, no. 4, pp. 734-744, Aug. 2011.

[35] Hao Jiang, Hongli Ji, Jinhao Qiu, and Yuansheng Chen, "A modified prandtl-ishlinskii model for modeling asymmetric hysteresis of piezoelectric actuators", IEEE Transactions on Ultrasonics, Ferroelectrics and Frequency Control, vol. 57, no. 5, pp. 1200-1210, May 2010.

[36] R. Bouc, "Forced vibration of mechanical systems with hysteresis", in Proceedings of Conference on Nonlinear Oscillations, Prague, Czechoslovakia, 1967, pp. 315-315.

[37] Y. K. Wen, "Method for random vibration of hysteresis systems", Journal of the Engineering Mechanics Division, vol. 102, no. 2, pp. 249-263, Apr. 1976.

[38] J. Song, and A. Der Kiureghian, " Generalized Bouc-Wen model for highly asymmetric hysteresis", Journal of engineering mechanics, vol. 132, no. 6, pp. 610-618, Jun. 2006.

[39] M. Rakotondrabe, "Bouc-Wen modeling and inverse multiplicative structure to compensate hysteresis nonlinearity in piezoelectric actuators", IEEE Transactions on Automation Science and Engineering, vol. 8, no. 2, pp. 428-431, Apr. 2011.

[40] I. D. Mayergoyz, "Mathematical models of hysteresis", IEEE Transactions on Magnetics, vol. 22, no. 5, pp. 603-608, Sept. 1986.

[41] M. d'Aquino, C. Serpico, C. Visone, A. A. Adly, "A new vector model of magnetic hysteresis based on a novel class of Play hysterons", IEEE Transactions on Magnetics, vol. 39, no. 5, pp. 2537-2539, Sept. 2003.

[42] Y. J. Park, Y. K. Wen, A. H-S. Ang, "Random vibration of hysteretic systems under bidirectional ground motions", Earthquake engineering structural dynamics, vol. 14, no. 4, pp. 543-557, Aug. 1986.

[43] T. S. Low, and W. Guo, "Modeling of a three-layer piezoelectric bimorph beam with hysteresis", Journal of Microelectromechanical Systems, vol. 4, no. 4, pp. 230-237, Dec. 1995.

[44] E. H. Moore, "On the reciprocal of the general algebraic matrix", Bulletin of the American Mathematical Society, vol. 26, pp. 394-395, 1920.

[45] T. Tuma, J. Lygeros, A. Sebastian, and A. Pantazi, "Optimal scan trajectories for high-speed scanning probe microscopy", in Proceedings of American Control Conference, Montreal, Canada, Jun. 2012, pp. 3791-3796. 